\title{
POLYNOMIAL INTERPOLATION IN HIGHER DIMENSION: FROM SIMPLICIAL COMPLEXES TO GC SETS
}

\author{
NATHAN FIELDSTEEL AND HAL SCHENCK
}

\begin{abstract}
Geometrically characterized (GC) sets were introduced by ChungYao in their work on polynomial interpolation in $\mathbb{R}^{d}$. Conjectures on the structure of GC sets have been proposed by Gasca-Maeztu for the planar case, and in higher dimension by de Boor and Apozyan-Hakopian. We investigate GC sets in dimension three or more, and show that one way to obtain such sets is from the combinatorics of simplicial complexes.
\end{abstract}

\section{INTRODUCTION}

Given a set of points $X \subseteq \mathbb{R}^{d}$, one goal of interpolation is to find a set of functions which separate the points; that is, so that for each point $p \in X$, there is a unique function which vanishes on $X \backslash p$ but not at $p$. Perhaps the most studied case occurs when the functions are polynomials.

Definition 1.1. [6] A set of points $X \subseteq \mathbb{R}^{d}$ is n-correct if the evaluation map on the set of polynomials of degree at most $n$ is an isomorphism onto $\mathbb{R}^{|X|}$; note that to be $n$-correct $X$ must consist of $\left(\begin{array}{c}d+n \\ n\end{array}\right)$ points. A k-dimensional affine subspace of $\mathbb{R}^{d}$ is maximal if it contains a subset of $X$ of cardinality $\left(\begin{array}{c}k+n \\ n\end{array}\right)$.

For example, a hyperplane is maximal if it contains a subset of $X$ of cardinality $\left(\begin{array}{c}d-1+n \\ n\end{array}\right)$. Chung-Yao introduced the geometrically characterized property:

Definition 1.2. [12], A set $X$ of $\left(\begin{array}{c}n+d \\ d\end{array}\right)$ points in $\mathbb{R}^{d}$ is called a $G C_{d, n}$ set if for each point $p \in X$, there exists a product

$$
Q_{p}=\prod_{k=1}^{n} l_{k}
$$

of linear forms $l_{k}$ such that $Q_{p}(q)=\delta_{p q}$ for all $p, q \in X$.

Clearly a $G C_{d, n}$ set is n-correct. In [16, Gasca-Maeztu conjectured that in $\mathbb{R}^{2}$, every $G C_{2, n}$ set contains a line with $n+1$ points of $X$, which is a maximal hyperplane. In [8], Busch shows the conjecture holds for $n \leq 4$. The last 30 years have seen much additional work on the conjecture; see [7, 9], 10, 11], [17, [18, 20], 22. In [9] Carnicer-Gasca showed that the Gasca-Maeztu conjecture implies that a $G C_{2, n}$ set in $\mathbb{R}^{2}$ contains 3 maximal lines. Building on this, in [6], de Boor proposed two generalizations of the Gasca-Maeztu conjecture:

Conjecture 1.3. $A G_{d, n}$ set contains a maximal hyperplane.

Conjecture 1.4. $A G C_{d, n}$ set contains at least $d+1$ maximal hyperplanes.

2000 Mathematics Subject Classification. Primary 41A05, Secondary 41A10, 41A65.

Key words and phrases. Polynomial interpolation, Simplicial complex, Bi-Cohen Macaulay.

Schenck supported by NSF 1312071. 


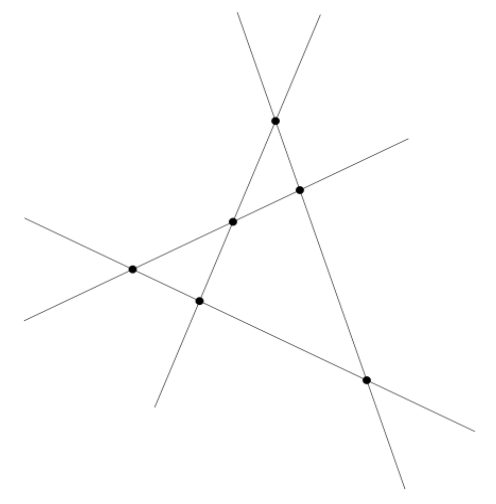

FiguRe 1. Four general lines in the plane, and their intersection points

de Boor shows that Conjecture 1.4 will require some additional hypothesis: he constructs a $G C_{3,2}$ set which does not have four maximal hyperplanes. Apozyan [1] used this to construct a $G C_{6,2}$ set with no maximal hyperplane, so Conjecture 1.3 fails as stated. On the other hand, 3 . shows Conjecture 1.3 holds for $G C_{3,2}$ sets. Apozyan-Hakopian conjecture in [1] that a $G C_{d, n}$ set contains at least $\left(\begin{array}{c}d+1 \\ 2\end{array}\right)$ maximal lines, which is proved for $d=3, n=2$ in [2. We study $G C_{d, n}$ sets, focussing mainly on the case $d \geq 3$ and $n \geq 2$. Our starting point is work of Sauer-Xu in [25] showing that the ideal $I_{X}$ of a $G C_{d, n}$ set $X$ is minimally generated in degree $n+1$ by $\left(\begin{array}{c}n+d \\ n+1\end{array}\right)$ products of linear forms.

The central idea of this paper is to lift the ideal $I_{X}$ of polynomials vanishing on $X$ to a monomial ideal: by replacing the generators $\prod l_{i}$ of $I_{X}$ with monomials $\prod y_{i}$ with a new variable $y_{i}$ for each distinct linear form, we obtain insight into the combinatorial structure of GC sets: the new monomial ideal is squarefree, so corresponds via Stanley-Reisner theory to a simplicial complex $\Delta$. The core of the paper is $\S 3$, where we apply Stanley-Reisner theory to analyze these ideals. Theorem 3.12 shows that Bi-Cohen Macaulay squarefree monomial ideals of codimension $d$ and degree $\left(\begin{array}{c}d+n \\ n\end{array}\right)$ always specialize to $n$-correct sets of points.

As our goal is to obtain examples of GC sets, we reverse engineer this process, by starting with a Bi-Cohen Macaulay monomial ideal. While specializing yields a $n$-correct set, the GC condition is quite restrictive: most $n$-correct sets are not GC. To overcome this obstacle, we introduce an analog of the GC property for monomial ideals. In Theorem 3.13, we prove a combinatorial criterion for a component of a monomial ideal to be GC. Example 1.5 below illustrates our results in the $d=2$ case; additional examples appear in $\S 4$.

Example 1.5. A Chung-Yao natural lattice of six points in $\mathbb{R}^{2}$ consists of the intersection points of four general lines $\left\{l_{1}, l_{2}, l_{3}, l_{4}\right\}$ in the plane. The ideal of $I_{X}=\left\langle l_{1} l_{2} l_{3}, l_{1} l_{2} l_{4}, l_{1} l_{3} l_{4}, l_{2} l_{3} l_{4}\right\rangle$, so replacing $l_{i}$ with $y_{i}$ gives rise to the ideal $I_{\Delta}=$ $\left\langle y_{1} y_{2} y_{3}, y_{1} y_{2} y_{4}, y_{1} y_{3} y_{4}, y_{2} y_{3} y_{4}\right\rangle$. The ideal $I_{\Delta}$ has a decomposition

$$
I_{\Delta}=\left\langle y_{1}, y_{2}\right\rangle \cap\left\langle y_{1}, y_{3}\right\rangle \cap\left\langle y_{1}, y_{4}\right\rangle \cap\left\langle y_{2}, y_{3}\right\rangle \cap\left\langle y_{2}, y_{4}\right\rangle \cap\left\langle y_{3}, y_{4}\right\rangle .
$$

The results in $\S 3$ show $\Delta$ consists of 4 vertices and 6 edges connecting them. 
For this example a component $F=\left\langle y_{i}, y_{j}\right\rangle$ in Equation 1 satisfies the monomial version of the GC condition appearing in Definition 3.9 if there is a quadratic monomial $f$ such that $f \notin F$ but $f \cdot F \in I_{\Delta}$. For example when $F=\left\langle y_{1}, y_{2}\right\rangle$, choosing $f=y_{3} y_{4}$ satisfies the condition, and an easy check shows for the other components $\left\langle y_{i}, y_{j}\right\rangle$ choosing $f=y_{k} y_{l}$ with $\{i, j, k, l\}=\{1,2,3,4\}$ works. Each of the hyperplanes $y_{i}$ appears in 3 of the $\left\langle y_{i}, y_{j}\right\rangle$; the $y_{i}$ are monomial versions of maximal hyperplanes. Specializing $y_{i} \mapsto l_{i}$ preserves these properties, and reproves the well known fact that a Chung-Yao configuration of $n+2$ lines in the plane is $G C_{2, n}$ and has $n+2$ maximal hyperplanes.

\section{The Vanishing ideal of A $G C_{d, n}$ SET}

In this section, we show that a set of points $X$ having the $G C_{d, n}$ property is very special from an algebraic standpoint. Recall that for a set of points $X$, the set of polynomial functions vanishing on all $p \in X$ is the vanishing ideal $I_{X}$ of $X$, and is closed under addition, as well as under multiplication by arbitrary polynomials.

The first step in our analysis of GC sets is to streamline the algebra by homogenizing the problem. Geometrically, this means we consider affine space $\mathbb{R}^{d}$ as a subset of projective space $\mathbb{P}_{\mathbb{R}}^{d}$. Since $\mathbb{P}_{\mathbb{R}}^{d}$ may be thought of as $\mathbb{R}^{d} \bigcup \mathbb{P}_{\mathbb{R}}^{d-1}$, where $\mathbb{P}_{\mathbb{R}}^{d-1}$ is the hyperplane at infinity and $X \subseteq \mathbb{R}^{d}, X \cap \mathbb{P}_{\mathbb{R}}^{d-1}=\emptyset$. We now demonstrate the utility of this construction.

Example 2.1. Suppose

$$
X=(0,0) \cup(1,0) \cup(0,1) \subseteq \mathbb{R}^{2} .
$$

$I_{X}$ consists of the intersections of the ideal of the three points, so is

$$
\left(x_{0}, x_{1}\right) \cap\left(x_{0}-1, x_{1}\right) \cap\left(x_{0}, x_{1}-1\right) .
$$

If we embed $\mathbb{R}^{2} \subseteq \mathbb{P}_{\mathbb{R}}^{2}$ as the plane with $x_{2}=1$, the points become

$(0: 0: 1) \cup(1: 0: 1) \cup(0: 1: 1)$, where the colon denotes projective coordinates; since projective points can scale by any $\lambda \in \mathbb{R}^{*}$, the corresponding ideal is $\left(x_{0}, x_{1}\right) \cap\left(x_{0}-x_{2}, x_{1}\right) \cap\left(x_{0}, x_{1}-x_{2}\right)=\left\langle x_{0} x_{1}, x_{0}\left(x_{0}-x_{2}\right), x_{1}\left(x_{1}-x_{2}\right)\right\rangle \subseteq \mathbb{R}\left[x_{0}, x_{1}, x_{2}\right]$.

Why do this? The answer is that the ideal $\left\langle x_{0} x_{1}, x_{0}\left(x_{0}-x_{2}\right), x_{1}\left(x_{1}-x_{2}\right)\right\rangle$ is determinantal, that is, the generators are the $2 \times 2$ minors of the matrix

$$
\left[\begin{array}{cc}
x_{0}-x_{2} & x_{1}-x_{2} \\
-x_{1} & 0 \\
0 & -x_{0}
\end{array}\right]
$$

This is not an accident: it can be shown that after homogenizing, any $G C_{2, n}$ set is generated by the maximal minors of a $n+2 \times n+1$ matrix of homogeneous linear forms. In Example 1.5, $I_{X}$ is generated by the $3 \times 3$ minors of

$$
d_{2}=\left[\begin{array}{ccc}
l_{4} & 0 & 0 \\
-l_{3} & l_{3} & 0 \\
0 & -l_{2} & l_{2} \\
0 & 0 & -l_{1}
\end{array}\right]
$$

However, there is even more structure here: the columns of the matrix $d_{2}$ are generators (over the polynomial ring) for the kernel of the matrix

$$
d_{1}=\left[\begin{array}{llll}
l_{1} l_{2} l_{3} & l_{1} l_{2} l_{4} & l_{1} l_{3} l_{4} & l_{2} l_{3} l_{4}
\end{array}\right] .
$$


Relations on a matrix with polynomial entries are called syzygies. They can be represented by a vector of polynomials, and were systematically studied by Hilbert. For a $G C_{2, n}$ set $X$, there are three points to highlight:

- The generators for $I_{X}$ are products of linear forms.

- The first syzygies of $I_{X}$ are generated by vectors of linear forms.

- The maximal minors of the syzygy matrix generate $I_{X}$.

The second two points are consequences of a famous theorem in commutative algebra, the Hilbert-Burch theorem, which describes the behavior of ideals which define sets of points in the projective plane. Most of the remainder of this section is devoted to defining these objects, and to understanding what happens for GC sets in higher dimensions.

By our earlier remarks, we may assume the GC set $X$ consists of points in $\mathbb{P}_{\mathbb{R}}^{d}$; $R$ will denote the ring $\mathbb{R}\left[x_{0}, \ldots, x_{d}\right]$. For a point $p \in X$,

$$
I_{p}=\left\langle l_{p, 1}, \ldots, l_{p, d}\right\rangle
$$

is generated by $d$ independent homogeneous linear forms. We use $\mathcal{Q}$ to denote the ideal $\left\langle Q_{p}, p \in X\right\rangle$, with $Q_{p}$ as in Definition 1.2 .

In algebraic geometry, a set of points $X$ imposes independent conditions on polynomials of degree $n$ if the rank of the evaluation map is equal to $|X|$. So an $n$-correct set in $\mathbb{R}^{d}$ is a set of $\left(\begin{array}{c}d+n \\ n\end{array}\right)$ points which imposes independent conditions in degree $n$. Let $X \subseteq \mathbb{P}^{d}$ be a set of $N=\left(\begin{array}{c}d+n \\ n\end{array}\right)$ distinct points having property $G C_{d, n}$.

Lemma 2.2. The ideal $\mathcal{Q}$ is of the form $\left\langle x_{0}, \ldots, x_{d}\right\rangle^{n}$.

Proof. Since the $Q_{p}$ are all of degree $n$, clearly $\mathcal{Q} \subseteq\left\langle x_{0}, \ldots, x_{d}\right\rangle^{n}$. The condition that $Q_{p}(q)=\delta_{p q}$ means that the $Q_{p}$ are linearly independent; since the dimension of $\left\langle x_{0}, \ldots, x_{d}\right\rangle^{n}$ is $\left(\begin{array}{c}n+d \\ d\end{array}\right)$, equality holds. this means $G C_{d, n}$ sets are $n$-correct.

Lemma 2.3. Suppose $X$ has the $G C_{d, n}$ property. Then for each $p \in X$, there are $d$ linearly independent linear forms $l_{p, 1}, \ldots, l_{p, d}$ with each $l_{p, j}$ dividing some $Q_{q}$, $p \neq q$, such that $l_{p, j}(p)=0$.

Proof. The $G C_{d, n}$ property implies that each $Q_{p}$ with $p \neq q$ has a factor which is a linear form passing thru $p$. Let $L$ be the vector space generated by all such linear factors, and suppose $L$ has dimension less than $d$. Changing coordinates, we can suppose $L=\left\{x_{0}, \ldots, x_{m}\right\}$ with $m \leq d-2$. But then

$$
\mathcal{Q}=\left\langle Q_{p}\right\rangle+\langle P\rangle
$$

where $\langle P\rangle=\mathcal{Q}_{n} \cap\left\langle x_{0}, \ldots, x_{m}\right\rangle$. This is impossible, because by Lemma 2.2, $\mathcal{Q}=$ $\left\langle x_{0}, \ldots, x_{d}\right\rangle^{n}$ and

$$
\left\{x_{d-1}^{n}, x_{d-1}^{n-1} x_{d}, \ldots, x_{d-1} x_{d}^{n-1}, x_{d}^{n}\right\} \subseteq \mathcal{Q}_{n}
$$

is $n+1$ dimensional and disjoint from the degree $n$ component of the subideal of $\mathcal{Q}$ generated by $\langle P\rangle$, and clearly cannot be spanned by $Q_{p}$.

2.1. Minimal free resolutions. The polynomial $\operatorname{ring} R=\mathbb{R}\left[x_{0}, \ldots, x_{d}\right]$ is a $\mathbb{Z}$-graded ring: $R_{i}$ is the vector space of homogeneous polynomials of degree $i$, and if $r_{j} \in R_{j}$ and $r_{i} \in R_{i}$ then $r_{i} \cdot r_{j} \in R_{i+j}$. As $R_{0}=\mathbb{R}$, this means each $R_{i}$ has the structure of an $R_{0}=\mathbb{R}$ vector space, of dimension $\left(\begin{array}{c}n+d \\ d\end{array}\right)$, and $R=\oplus_{i} R_{i}$. A finitely generated graded $R$-module $N$ admits a similar decomposition; if $s \in R_{p}$ 
and $n \in N_{q}$ then $s \cdot n \in N_{p+q}$. In particular, each $N_{q}$ is a $R_{0}=\mathbb{R}$-vector space. A graded map of graded modules $M \rightarrow N$ preserves the grading, so takes $M_{i} \rightarrow N_{i}$.

Definition 2.4. For a finitely generated graded $S$-module $N$, the Hilbert function is $H F(N, t)=\operatorname{dim}_{\mathbb{R}} N_{t}$, and the Hilbert series is $H S(N, t)=\sum \operatorname{dim}_{\mathbb{R}} N_{q} t^{q}$.

For $t \gg 0$, the Hilbert function of $N$ is a polynomial in $t$, called the Hilbert polynomial $H P(N, t)$, of degree at most $d$ ([26], Theorem 2.3.3). For $X \subseteq \mathbb{P}^{d}$, we define $\operatorname{codim}\left(I_{X}\right)$ as $d-\operatorname{deg}\left(H P\left(R / I_{X}, t\right)\right)$. The degree of $H P\left(R / I_{X}, t\right)$ is the dimension of $X$. When $X$ is a set of points in $\mathbb{P}^{d}, I_{X}=\cap P_{i}$ with $P_{i}=\left\langle l_{i 1}, \ldots, l_{i d}\right\rangle$ and the codimension of $I_{X}$ is $d$.

Definition 2.5. A free resolution for an $R$-module $N$ is an exact sequence

$$
\mathbb{F}: \cdots \rightarrow F_{i} \stackrel{d_{i}}{\rightarrow} F_{i-1} \rightarrow \cdots \rightarrow F_{0} \rightarrow N \rightarrow 0,
$$

where the $F_{i}$ are free $R$-modules.

If $N$ is graded, then the $F_{i}$ are also graded, so letting $R(-m)$ denote a rank one free module generated in degree $m$, we may write $F_{i}=\oplus_{j} R(-j)^{a_{i, j}}$. By the Hilbert syzygy theorem [26] a finitely generated, graded $R$-module $N$ has a free resolution of length at most $d+1$, with all the $F_{i}$ of finite rank. Since

$$
\begin{aligned}
& H S(R(-i), t)=\frac{t^{i}}{(1-t) d+1} \\
& H P(R(-i), t)=\left(\begin{array}{c}
t+d-i \\
d
\end{array}\right)
\end{aligned}
$$

this means we can read off the Hilbert series, function and polynomial from a free resolution as an alternating sum, which is illustrated in Example 2.7

Definition 2.6. For a finitely generated graded $R$-module $N$, a free resolution is minimal if for each $i, \operatorname{Im}\left(d_{i}\right) \subseteq \mathfrak{m} F_{i-1}$, where $\mathfrak{m}=\left\langle x_{0}, \ldots, x_{d}\right\rangle$. The CastelnuovoMumford regularity of $N$ is $\max _{i, j}\left\{a_{i, j}-i\right\}$. The projective dimension $\operatorname{pdim}(N)$ of $N$ is the length of a minimal free resolution of $N$.

Example 2.7. For the $R=\mathbb{R}\left[x_{0}, x_{1}, x_{2}\right]$ module $R /\left\langle x_{0}^{2}, x_{1}^{2}\right\rangle$, the graded free resolution is

$$
0 \longrightarrow R(-4) \stackrel{\left[\begin{array}{c}
-x_{1}^{2} \\
x_{0}^{2}
\end{array}\right]}{\longrightarrow} R(-2)^{2} \stackrel{\left[\begin{array}{ll}
x_{0}^{2} & x_{1}^{2}
\end{array}\right]}{\longrightarrow} R \longrightarrow R / I \longrightarrow 0,
$$

and for $I_{X}$ of Example 1.5 the free resolution is

$$
0 \rightarrow R(-4)^{3} \stackrel{d_{2}}{\rightarrow} R^{4}(-3) \stackrel{d_{1}}{\rightarrow} R \rightarrow R / I \rightarrow 0,
$$

with $d_{i}$ as in Example 2.1; the $d_{1}$ map is a $1 \times 4$ matrix with cubic entries, giving a map $R^{4} \rightarrow R^{1}$. Because we want graded maps, the generators of $R^{4}$ must appear in degree 3 , explaining the module $R^{4}(-3)$. So for $X$ the Chung-Yao set of Example 1.5. we see that the Hilbert series and Hilbert polynomial are

$$
\begin{array}{ccc}
H S\left(R / I_{X}, t\right) & = & \frac{1-4 t^{3}+3 t^{4}}{(1-t)^{3}} \\
H P\left(R / I_{X}, t\right) & = & \left(\begin{array}{c}
t+2 \\
2
\end{array}\right)-4\left(\begin{array}{c}
t+2-3 \\
2
\end{array}\right)+3\left(\begin{array}{c}
t+2-4 \\
2
\end{array}\right)=6,
\end{array}
$$

as expected, since the Hilbert polynomial of a $G C_{d, n}$ set $X$ is $|X|=\left(\begin{array}{c}d+n \\ n\end{array}\right)$.

While the differentials which appear in a minimal free resolution of $N$ are not unique, the ranks and degrees of the free modules which appear are unique. 
Definition 2.8. An ideal $I \subseteq R$ is Cohen-Macaulay if $\operatorname{codim}(I)=\operatorname{pdim}(R / I)$.

Example 2.9. The two ideals in Example 2.7 both have $\operatorname{pdim}(R / I)=2$; because the ideals define zero dimensional subsets of the plane they are codimension two, so both ideals are Cohen-Macaulay. This is a general phenomenon: the ideal $I_{X}$ of a set of points $X \subseteq \mathbb{P}^{d}$ is Cohen-Macaulay, of codimension $d$.

Definition 2.8 is hard to digest, but the Cohen-Macaulay condition has many useful consequences, see Chapter 10 of 26. The Hilbert-Burch theorem states that a codimension two Cohen-Macaulay ideal $\mathcal{I}=\left\langle f_{1}, \ldots, f_{m}\right\rangle$ is generated by the maximal minors of an $m \times m-1$ matrix, whose columns are a basis for the syzygies on $\mathcal{I}$. To generalize the Hilbert-Burch theorem to codimension greater than two, we need the Eagon-Northcott complex:

Definition 2.10. Let $R^{m} \simeq F \stackrel{\phi}{\rightarrow} G \simeq R^{n}$ be a homomorphism of $R$-modules, with $m \geq n$. Then $\phi$ induces a homomorphism

$$
\Lambda^{n}(F) \stackrel{\Lambda \phi}{\longrightarrow} \Lambda^{n}(G)=R,
$$

where the entries of $\Lambda \phi$ are the $n \times n$ minors of $\phi$. With suitable conditions (see 23] ) on $\phi$, the ideal $I_{\phi}$ of $n \times n$ minors has a minimal free resolution, in which the free modules are tensor products of exterior and symmetric powers:

$\cdots \longrightarrow S_{2}\left(G^{*}\right) \otimes \Lambda^{n+2}(F) \longrightarrow S_{1}\left(G^{*}\right) \otimes \Lambda^{n+1}(F) \stackrel{d_{1}}{\longrightarrow} \Lambda^{n}(F) \longrightarrow R \longrightarrow R / I_{\phi} \longrightarrow 0$.

The key map is $d_{1}$ : since $\phi^{*}: G^{*} \rightarrow F^{*}$, for $\alpha \in G^{*}, \phi^{*}(\alpha) \in F^{*}$, and

$$
d_{1}\left(\alpha \otimes e_{1} \wedge \cdots \wedge e_{n+1}\right)=\sum_{j=1}^{n+1}(-1)^{j}\left(\phi^{*}(\alpha)\left(e_{j}\right)\right) \cdot e_{1} \wedge \cdots \wedge \widehat{e_{j}} \wedge \cdots \wedge e_{n+1},
$$

with higher differentials defined similarly.

2.2. The ideal of a $G C_{d, n}$ set is generated by products of linear forms. We start with an algebraic proof of the following key result of Sauer-Xu 25], which is a main ingredient in this paper.

Theorem 2.11. If $X \subseteq \mathbb{R}^{d}$ is a $G C_{d, n}$ set, then the ideal $I_{X}$ is generated in degree $n+1$ by $\left(\begin{array}{l}n+d \\ n+1\end{array}\right)$ products of linear forms.

Proof. Let $I_{C}=\left\langle Q_{p} \cdot l_{p j}, p \in X, j \in\{1, \ldots, d\}\right\rangle$, with $l_{p, j}$ as in Lemma 2.3. Because $X$ is a set of distinct points in $\mathbb{P}^{d}, I_{X}$ is Cohen-Macaulay and codimension $d$. Since $Q_{p}(q)=\delta_{p q}$, the points of $X$ impose independent conditions (see [26, Chapter 7) on polynomials of degree $n$, so $I_{X}$ is generated in degree $>n$. As $\operatorname{dim}_{\mathbb{R}} R_{n+1}=\left(\begin{array}{c}n+1+d \\ d\end{array}\right)$ and the $\left(\begin{array}{c}n+d \\ d\end{array}\right)$ points impose independent conditions, by Theorem 7.1.8 of [26], $I_{X}$ is generated by

$$
\left(\begin{array}{c}
n+1+d \\
d
\end{array}\right)-\left(\begin{array}{c}
n+d \\
d
\end{array}\right)=\left(\begin{array}{l}
n+d \\
n+1
\end{array}\right)
$$

polynomials of degree $n+1$.

By construction, every polynomial in $I_{C}$ is a product of linear forms of degree $n+1$ and vanishes on $X$, so $I_{C} \subseteq I_{X}$. It suffices to show that the dimension of $I_{C}$ in degree $n+1$ is $\left(\begin{array}{l}n+d \\ n+1\end{array}\right)$. There are relations among the generators of $I_{C}$ :

$$
\sum_{i=1}^{N} Q_{i}\left(\sum_{j=1}^{d} a_{i_{j}} l_{i_{j}}\right)=0
$$


with the $a_{i_{j}} \in \mathbb{R}$. Such a relation is a linear syzygy on $\mathcal{Q}=\left\langle x_{0}, \ldots, x_{d}\right\rangle^{n}$. By [14], $\mathcal{Q}$ has a minimal free resolution of Eagon-Northcott type; in particular, $\mathcal{Q}$ is generated by the $n \times n$ minors of an $(n+d) \times n$ matrix whose entries are the variables of $R$. As a consequence, all linear syzygies are Eagon-Northcott type syzygies, that is, the image of the leftmost map below:

$$
S_{1}\left(R^{n}\right) \otimes \Lambda^{n+1}\left(R^{n+d}\right) \longrightarrow \Lambda^{n}\left(R^{n+d}\right) \longrightarrow \Lambda^{n}\left(R^{n}\right)=R \longrightarrow R / \mathcal{Q} \longrightarrow 0 .
$$

So there are $n \cdot\left(\begin{array}{l}n+d \\ n+1\end{array}\right)$ minimal linear first syzygies on $\mathcal{Q}$. The minimal value for $\operatorname{dim}\left(I_{C}\right)_{n+1}$ is achieved if these syzygies occur in Equation 2, so

$$
\begin{array}{rlc}
\operatorname{dim}\left(I_{C}\right)_{n+1} & \geq & d \cdot N-n \cdot\left(\begin{array}{l}
n+d \\
n+1
\end{array}\right) \\
& = & d \cdot\left(\begin{array}{c}
n+d \\
n
\end{array}\right)-n \cdot\left(\begin{array}{l}
n+d \\
n+1
\end{array}\right) \\
& = & \left(\begin{array}{l}
n+d \\
n+1
\end{array}\right) \\
& = & \operatorname{dim}\left(I_{X}\right)_{n+1}
\end{array}
$$

Since $I_{C} \subseteq I_{X}$ and both are generated in degree $n+1$, we have $I_{C}=I_{X}$.

An important related result is the next proposition; while the proof is technical the meaning is very concrete: if $X$ is a $G C_{d, n}$ set, then all the matrices in the minimal free resolution have entries of degree at most one: that is, they are matrices of linear forms, just as in the case where $d=2$.

Proposition 2.12. The minimal free resolution of $I_{X}$ has the same graded free modules as an Eagon-Northcott resolution of a generic $(n+d) \times(n+1)$ matrix.

Proof. By Theorem 7.1.8 of [26], the Castelnuovo-Mumford regularity of $I_{X}$ is the smallest $i$ such that $H^{1}\left(\mathcal{I}_{X}(i-1)\right)=0$; because the points impose independent conditions and $H^{1}\left(\mathcal{I}_{X}(i-1)\right)$ is the cokernel of the evaluation map on polynomials of degree $i-1$, the $G C_{d, n}$ property means $X$ is $n+1$ regular. Therefore the minimal free resolution of $I_{X}$ has the form

$$
0 \rightarrow R^{a_{d}}(-d-n) \rightarrow R^{a_{d-1}}(-d-n+1) \rightarrow \cdots R^{a_{1}}(-n-1) \rightarrow R \rightarrow R / I_{X} \rightarrow 0,
$$

so every differential is a matrix of linear forms. Since the points impose independent conditions, comparing to the Hilbert series yields the result.

Definition 2.13. We call an ideal $I$ determinantal if $I$ is generated by the $r \times r$ minors of a $m \times r$ matrix, with $m \geq r \geq 2$.

Example 2.14. For a set of points $X \subseteq \mathbb{P}^{2}$, the Hilbert-Burch theorem [23] shows that $I_{X}$ is determinantal, with $m=n+2, r=n+1$. This fails in higher dimension: the ideal for ten general points in $\mathbb{P}^{3}$ has a minimal free resolution of the form

$$
0 \longrightarrow R(-5)^{6} \longrightarrow R(-4)^{15} \longrightarrow R(-3)^{10} \longrightarrow R \longrightarrow R / I \longrightarrow 0 \text {. }
$$

So $I_{X}$ has 10 cubic generators, 15 linear first syzygies, and 6 linear second syzygies. However, it is not determinantal [19. By Proposition 2.12 the graded free modules are the same as those of a $G C_{3,2}$ set; by Theorem $3.12 I_{X}$ is determinantal if $X$ is Chung-Yao. Question: are $G C_{d, n}$ sets always determinantal?

\section{Bi-Cohen Macaulay simplicial complexes}

By Theorem 2.11, the ideal $I_{X}$ can be generated by products of linear forms, and our strategy is to relate $I_{X}$ to a monomial ideal. Because the forms appearing in any generator $F$ of $I_{X}$ are distinct, the monomial ideal is actually squarefree. Such ideals are related to the combinatorics of simplicial complexes. 


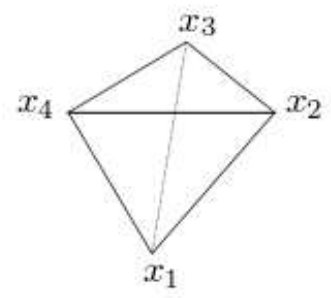

Figure 2. One skeleton of a three simplex

\subsection{Simplicial complexes and Stanley-Reisner ring.}

Definition 3.1. 26] A simplicial complex $\Delta$ on a vertex set $V$ is a collection of subsets $\sigma$ of $V$, such that if $\sigma \in \Delta$ and $\tau \subset \sigma$, then $\tau \in \Delta$. If $|\sigma|=i+1$ then $\sigma$ is called an $i-$ face.

Let $f_{i}(\Delta)$ be the number of $i$-faces of $\Delta$, and $\operatorname{dim}(\Delta)=\max \left\{i \mid f_{i}(\Delta) \neq 0\right\}$. If $\operatorname{dim}(\Delta)=n-1$, let $f_{\Delta}(t)=\sum_{i=0}^{n} f_{i-1} t^{n-i}$, with $f_{-1}=1$ for the empty face. The reverse ordered list of coefficients of $f_{\Delta}(t)$ is the $f$-vector $f(\Delta)$ of $\Delta$.

Definition 3.2. The Alexander dual $\Delta^{\vee}$ of $\Delta$ is the simplicial complex

$$
\Delta^{\vee}=\left\{\tau^{\vee} \mid \tau \notin \Delta\right\} \text {, where } \tau^{\vee} \text { denotes the complement } V \backslash \tau \text {. }
$$

Definition 3.3. Let $\Delta$ be a simplicial complex on vertices $\left\{y_{1}, \ldots, y_{n}\right\}$. The Stanley-Reisner ideal $I_{\Delta}$ is

$$
\left.I_{\Delta}=\left\langle y_{i_{1}} \cdots y_{i_{j}}\right|\left\{y_{i_{1}}, \ldots, y_{i_{j}}\right\} \text { is not a face of } \Delta\right\rangle \subseteq S=\mathbb{R}\left[y_{1}, \ldots y_{n}\right] \text {, }
$$

and the Stanley-Reisner ring is $\mathbb{R}\left[y_{1}, \ldots y_{n}\right] / I_{\Delta}$.

The Stanley-Reisner ideal $I_{\Delta} \vee$ of $\Delta^{\vee}$ is obtained by monomializing the primary decomposition of $I_{\Delta}$ : for each primary component $P_{i}$ in the primary decomposition, take the product of the terms in the component. So if

$$
I_{\Delta}=\bigcap_{j} P_{j} \text { with } P_{j}=\left\langle y_{j_{1}}, \ldots, y_{j_{d}}\right\rangle
$$

then the minimal generators of $I_{\Delta} \vee$ are of the form $y_{j_{1}} \cdots y_{j_{d}}$.

Definition 3.4. The $j-1$ skeleton of a $i-1$ simplex has as maximal faces all $j$ tuples on a set of $i$ vertices. Denote this complex by $\Delta(i, j)$. The Stanley-Reisner ideal $I_{\Delta(i, j)}$ is generated by all square-free monomials of degree $j+1$ in $i$ variables.

Example 3.5. Figure 2 shows $\Delta(4,2)$. $\Delta$ consists of 4 vertices and 6 edges, so $\Delta=\left\{\emptyset,\left\{x_{i}\right\},\left\{x_{i}, x_{j}\right\} \mid 1 \leq i \leq 4\right.$ and $\left.i<j \leq 4\right\}$ and $f(\Delta)=(1,4,6)$. Every maximal nonface of $\Delta$ is a triangle, so $I_{\Delta}=\left\langle x_{1} x_{2} x_{3}, x_{1} x_{2} x_{4}, x_{1} x_{3} x_{4}, x_{2} x_{3} x_{4}\right\rangle$. The complements of the four triangles are the four vertices, so $\Delta^{\vee}=\Delta(4,1)$, the four vertices. Specializing $x_{i} \mapsto l_{i}$ yields the Chung-Yao set of Example 1.5.

Definition 3.6. A regular sequence on $S / I$ is a sequence $\left\{f_{1}, f_{2}, \ldots, f_{k}\right\} \subseteq S=$ $\mathbb{R}\left[y_{1}, \ldots, y_{n}\right]$ such that each $f_{i}$ is not a zero divisor on $S^{\prime}=S /\left(I, f_{1}, \ldots, f_{i-1}\right)$; alternatively, the map $S^{\prime} \stackrel{\cdot f_{i}}{\rightarrow} S^{\prime}$ is injective. The depth of $S / I$ is the length of a maximal regular sequence. It is a theorem [26] that the Cohen-Macaulay condition is equivalent to $\operatorname{depth}(S / I)=n-\operatorname{codim}(I)$. 
3.2. The simplicial complex of a GC set. We return to the study of $I_{X}$. Let $S=\mathbb{R}\left[y_{1}, \ldots, y_{m}\right]$, with a variable for each distinct (ignore scaling) linear form which is a factor of one of the $\prod l_{i}$ which generate $I_{X}$, and let $\phi: S \rightarrow R$ via $y_{i} \mapsto l_{i}$. The kernel $L$ of $\phi$ is an ideal generated by $m-d-1$ linear forms. Let $I^{\prime}$ be the ideal in $S$ obtained by substituting $y_{i}$ for $l_{i}$ in $I_{X}$, so $\phi$ induces a surjective map $\psi: S \stackrel{\phi}{\rightarrow} R \stackrel{\pi}{\rightarrow} R / I_{X}$. Since

$$
\frac{S}{I^{\prime}+L} \simeq \frac{S / L}{\left(I^{\prime}+L\right) / L} \simeq \frac{\phi(S)}{\phi\left(I^{\prime}\right)} \simeq \frac{R}{I_{X}}=R / \cap_{i=1}^{|X|}\left\langle l_{i_{1}}, \ldots, l_{i_{d}}\right\rangle,
$$

$I^{\prime}+L=\operatorname{ker}(\psi)$. Let $J=\cap_{i=1}^{|X|}\left\langle y_{i_{1}}, \ldots, y_{i_{d}}\right\rangle$. If $J$ has $\left(\begin{array}{c}n+d \\ n+1\end{array}\right)$ generators in degree $n+1$ then $I^{\prime}+L=J+L$ with $J$ a codimension $d$ squarefree monomial ideal. Since $S /(J+L) \simeq R / I_{X}, J+L$ is of codimension $m-1$ and depth one, the $m-d-1$ linear forms of $L$ are a regular sequence on $S / J$; because $\operatorname{depth}(S /(J+L))=1$, we can find an additional nonzero divisor on $S /(J+L)$. Thus $S / J$ has depth $m-d$ so is Cohen-Macaulay.

Definition 3.7. For a $G C_{d, n}$ set $X$ with defining ideal $I_{X}$, write $J_{\Delta(X)}$ for the squarefree monomial ideal $J$ appearing above, with $\Delta(X)$ the simplicial complex.

Theorem 3.8. If $I^{\prime}=J$ then the ideal $J_{\Delta(X)}$ is Bi-Cohen Macaulay: both $J_{\Delta(X)}$ and the Alexander dual $J_{\Delta(X) \vee}$ are Cohen-Macaulay.

Proof. The Eagon-Reiner theorem [13] states that a Stanley-Reisner ideal $I_{\Delta}$ is Cohen-Macaulay iff the Alexander dual ideal $I_{\Delta} v$ has a minimal free resolution where all the matrices representing the maps have only linear forms as entries. The remarks above show that if $I^{\prime}=J_{\Delta(X)}$ then the ideal is Cohen-Macaulay and by Proposition 2.12 has a linear minimal free resolution, so the result follows.

Even in algebraic geometry, Bi-Cohen Macaulay simplicial complexes are esoteric objects. In [15], Fløystad-Vatne note that if $\Delta$ is a simplicial complex on $m$ vertices, then the face vectors of $\Delta$ and $\Delta^{\vee}$ satisfy the relation

$$
f_{i}\left(\Delta^{\vee}\right)+f_{m-i-2}(\Delta)=\left(\begin{array}{c}
m \\
i+1
\end{array}\right) .
$$

Since $J_{\Delta(X) \vee}$ has $\left(\begin{array}{c}n+d \\ d\end{array}\right)$ generators in degree $d$, letting $i^{*}=m-i-2$ we have

\begin{tabular}{|c|c|c|c|c|c|c|c|c|c|c|c|}
\hline$i$ & 0 & 1 & 2 & $\cdots$ & $n-1$ & $n$ & $\cdots$ & $m-d-1$ & $\cdots$ & $m-3$ & $m-2$ \\
\hline$\left(\begin{array}{c}m \\
i+1\end{array}\right)$ & $m$ & $\left(\begin{array}{c}m \\
2\end{array}\right)$ & $\left(\begin{array}{c}m \\
3\end{array}\right)$ & $\cdots$ & $\left(\begin{array}{c}m \\
n\end{array}\right)$ & $\left(\begin{array}{c}m \\
n+1\end{array}\right)$ & $\cdots$ & $\left(\begin{array}{c}m \\
d\end{array}\right)$ & $\cdots$ & $\left(\begin{array}{c}m \\
2\end{array}\right)$ & $m$ \\
\hline$f_{i}(\Delta)$ & $m$ & $\left(\begin{array}{c}m \\
2\end{array}\right)$ & $\left(\begin{array}{c}m \\
3\end{array}\right)$ & $\cdots$ & $\left(\begin{array}{c}m \\
n\end{array}\right)$ & $\left(\begin{array}{c}m \\
n+1\end{array}\right)-\left(\begin{array}{c}n+d \\
n+1\end{array}\right)$ & $\cdots$ & $\left(\begin{array}{c}n+d \\
d\end{array}\right)$ & $\cdots$ & 0 & 0 \\
\hline$f_{i^{*}}\left(\Delta^{\vee}\right)$ & 0 & 0 & 0 & $\cdots$ & 0 & $\left(\begin{array}{c}n+d \\
n+1\end{array}\right)$ & $\cdots$ & $\left(\begin{array}{c}m \\
d\end{array}\right)-\left(\begin{array}{c}n+d \\
d\end{array}\right)$ & $\cdots$ & $\left(\begin{array}{c}m \\
2\end{array}\right)$ & $m$ \\
\hline
\end{tabular}

Proposition 3.1 of [15] gives a complete characterization of the $f$-vectors that are possible if $\Delta$ is Bi-Cohen Macaulay: any such $f$-vector is of the form

$$
(1+t)^{i} \cdot\left(1+m t+\left(\begin{array}{c}
m \\
2
\end{array}\right) t^{2}+\cdots+\left(\begin{array}{c}
m \\
k
\end{array}\right) t^{k}\right) .
$$

The key definition of this paper is a version of the GC property for monomial ideals:

Definition 3.9. Let $I_{\Delta}$ be a squarefree Bi-Cohen Macaulay monomial ideal of codimension $d$ and degree $\left(\begin{array}{c}n+d \\ d\end{array}\right)$. A primary component $P$ of $I_{\Delta}$ is monomial GC if there is a degree $n$ monomial $f$ with $f \in I_{\Delta}: P$ and $f \notin P$. If every primary component $P$ of $I_{\Delta}$ is monomial $G C$, then $I_{\Delta}$ is a monomial $G C_{d, n}$ ideal. $V\left(y_{i}\right)$ is a maximal monomial hyperplane if $V\left(y_{i}\right)$ contains $\left(\begin{array}{c}n+d-1 \\ d-1\end{array}\right)$ components of $V\left(I_{\Delta}\right)$. 
3.3. The simplicial complex of a Chung-Yao set. In certain cases, the $G C_{d, n}$ property is a consequence of combinatorics: it is inherited from a monomial $G C_{d, n}$ ideal. Suppose there is no overlap between the nonzero entries of $f\left(\Delta^{\vee}\right)$ and $f(\Delta)$ :

$$
f_{i}\left(\Delta^{\vee}\right) \cdot f_{m-i-2}(\Delta)=0 \text { for all } i .
$$

As $d \geq 3$ and $n \geq 2$, the assumption above implies that

$$
\left(\begin{array}{c}
m \\
d
\end{array}\right)-\left(\begin{array}{c}
n+d \\
d
\end{array}\right)=0, \text { so } m=n+d
$$

Lemma 3.10. If $f_{j}\left(\Delta^{\vee}\right) \cdot f_{m-j-2}(\Delta)=0$ for all $j$, then $J_{\Delta}=I_{\Delta(d+n, n)}$

Proof. By our observation above, $f_{j}\left(\Delta^{\vee}\right) \cdot f_{m-j-2}(\Delta)=0$ for all $\mathrm{j}$ implies that $m=n+d$. Therefore $i=0$ in Equation 4 , so $\Delta=\Delta(m, n)$, with $m=d+n$. By Theorem 2.11 $J_{\Delta}$ has $\left(\begin{array}{c}m \\ n+1\end{array}\right)=\left(\begin{array}{c}n+d \\ n+1\end{array}\right)$ generators, which is exactly the number of squarefree monomials of degree $n+1$ on $n+d$ vertices, hence $J_{\Delta}=I_{\Delta(d+n, n)}$.

In Lemma 2.8 of [19], Gorla shows that the ideal $I_{\Delta(d+n, n)}$ is determinantal, and has an Eagon-Northcott resolution. The construction is as follows: take an $(n+d) \times(n+1)$ matrix $M$ of constants, with no minor vanishing. Let $M^{\prime}$ be the result of multiplying the $i^{\text {th }}$ column of $M$ by the variable $y_{i}$. Then

$$
I_{n+1}\left(M^{\prime}\right)=I_{\Delta(d+n, n)}
$$

The primary decomposition of $I_{\Delta(d+n, n)}$ is straightforward. Because $\Delta(d+n, n)$ consists of all $n$ tuples on a groundset of size $n+d$,

$$
I_{\Delta(d+n, n)}=\bigcap_{1 \leq i_{1}<i_{2}<\cdots<i_{d} \leq n+d}\left\langle y_{i_{1}}, \ldots, y_{i_{d}}\right\rangle .
$$

For any of the coordinate hyperplanes $y_{i}$, it is clear that there are $\left(\begin{array}{c}n+d-1 \\ d-1\end{array}\right)$ terms in the primary decomposition which contain the fixed linear form $y_{i}$. For each component in the primary decomposition, $V\left(\left\langle y_{i_{1}}, \ldots, y_{i_{d}}\right\rangle\right)$ is a codimension $d$ linear subspace, and the count above shows that every coordinate hyperplane contains $\left(\begin{array}{c}n+d-1 \\ d-1\end{array}\right)$ such components of $V\left(I_{\Delta(d+n, n)}\right)$.

Theorem 3.11. If $I_{\Delta}$ is a squarefree Bi-Cohen Macaulay monomial ideal of codimension $d$ and degree $\left(\begin{array}{c}n+d \\ d\end{array}\right)$, then a specialization by a regular sequence $\phi: y_{i} \mapsto l_{i}$ yields a n-correct set. If in addition $I_{\Delta}$ is a monomial $G C_{d, n}$ ideal, then the specialization is also a $G C_{d, n}$ set. If $I_{\Delta}$ has a maximal hyperplane, so does $\phi\left(I_{\Delta}\right)$.

Proof. As $I_{\Delta}$ is Cohen-Macaulay, specialization by a regular sequence preserves the primary decomposition, hence the $G C_{d, n}$ and maximal hyperplane properties. The fact that the specialization is $n$-correct follows because specializing by a regular sequence preserves the minimal free resolution, and Proposition 2.12

Continuing with the example where $m=d+n$, for $m$ generic linear forms $l_{i} \in R$,

$$
\mathbb{R}\left[y_{1}, \ldots, y_{m}\right] \stackrel{\phi}{\longrightarrow} \mathbb{R}\left[x_{1}, \ldots, x_{d}\right], y_{i} \mapsto l_{i}
$$

yields the $G C_{d, n}$ sets of [12, which contain $n+d$ maximal hyperplanes. The argument above shows that they also have additional algebraic structure:

Theorem 3.12. If $X$ is a $G C_{d, n}$ set of Chung-Yao type, then $I_{X}$ is determinantal. 
3.4. Constructing GC sets from $I_{\Delta}$. One way to construct $G C_{d, n}$ sets is to start with a squarefree Bi-Cohen Macaulay monomial ideal of codimension $d$ and degree $\left(\begin{array}{c}n+d \\ d\end{array}\right)$, which is not a $G C_{d, n}$ monomial ideal, but which has many GC components. Any specialization will preserve the GC properties; if $I_{\Delta}$ has a maximal monomial hyperplane, specialization also preserves it. The next theorem is crucial: it gives a necessary and sufficient combinatorial condition for a primary component to be monomial GC:

Theorem 3.13. Let $I_{\Delta}$ be a squarefree Bi-Cohen Macaulay monomial ideal of degree $\left(\begin{array}{c}n+d \\ d\end{array}\right)$ and codimension $d$ :

$$
\left.I_{\Delta}=\bigcap_{i=1}^{\substack{n+d \\ d}}\right) P_{i}, \text { with } P_{i}=\left\langle x_{i_{1}}, \ldots, x_{i_{d}}\right\rangle \subseteq k\left[x_{1}, \ldots, x_{m}\right]
$$

A primary component $P_{i}=\left\langle x_{i_{1}}, \ldots, x_{i_{d}}\right\rangle$ is monomial GC iff there is $\tau \in \Delta_{n-1}$ such that for all $j \in\{1, \ldots, d\}, \overline{\tau v_{i_{j}}} \notin \Delta_{n}$, where $\overline{\tau v_{i_{j}}}$ is the join of $\tau$ with $v_{i_{j}}$.

Proof. From Definition 3.9, a primary component $P$ of $I_{\Delta}$ is monomial GC if there is a degree $n$ monomial (necessarily squarefree) $f$ with $f \in I_{\Delta}: P_{i}$ and $f \notin P_{i}$. As $I_{\Delta}$ is generated in degree $n+1, \Delta$ contains the $n-1$ skeleton $\Delta(m, n)$; in particular, $f$ corresponds to a face $\tau \in \Delta_{n-1}$. But $f \in I_{\Delta}: P_{i}$ iff $f \cdot x_{i_{k}} \in I_{\Delta}$ for all $k \in\{1, \ldots, d\}$ iff for all $j \in\{1, \ldots, d\}, \overline{\tau v_{i_{j}}} \notin \Delta$. Finally, the monomial $f$ is in $P_{i}$ iff for some $j \in\{1, \ldots, d\}, x_{i_{j}} \mid f$, which would imply there is a nonsquarefree monomial generator of $I_{\Delta}$, a contradiction.

\section{EXAmples}

We close with a pair of three dimensional examples.

Example 4.1. Consider the integral points of the tetrahedron with vertices at

$$
(0,0,0),(2,0,0),(0,2,0),(0,0,2)
$$

This is a Berzolari-Radon configuration of ten points in $\mathbb{R}^{3}$. Lifting to $\mathbb{P}^{3}$, we find the ideal $I_{X}$ is generated by the ten products of linear forms below:

$$
\begin{array}{cc}
x_{2} \cdot\left(x_{2}-x_{3}\right) \cdot\left(x_{2}-2 \cdot x_{3}\right), & x_{1} \cdot x_{2} \cdot\left(x_{2}-x_{3}\right), \\
\left(x_{2}-x_{3}\right) \cdot x_{2} \cdot x_{0}, & x_{1} \cdot x_{2} \cdot\left(x_{1}-x_{3}\right), \\
x_{0} \cdot\left(x_{0}-x_{3}\right) \cdot\left(x_{0}-2 \cdot x_{3}\right) & x_{0} \cdot x_{2} \cdot\left(x_{0}-x_{3}\right), \\
x_{1} \cdot\left(x_{1}-x_{3}\right) \cdot\left(x_{1}-2 \cdot x_{3}\right), & x_{0} \cdot x_{1} \cdot\left(x_{1}-x_{3}\right) \\
x_{0} \cdot x_{1} \cdot\left(x_{0}-x_{3}\right), & x_{0} \cdot x_{1} \cdot x_{2} .
\end{array}
$$

We replace this with the monomial ideal $J_{\Delta}$ generated by ten cubics:

$$
\begin{array}{ll}
y_{2} y_{6} y_{7}, & y_{1} y_{2} y_{6}, \\
y_{0} y_{2} y_{6}, & y_{1} y_{2} y_{5}, \\
y_{0} y_{4} y_{9} & y_{0} y_{2} y_{4}, \\
y_{1} y_{5} y_{8}, & y_{0} y_{1} y_{5}, \\
y_{0} y_{1} y_{4}, & y_{0} y_{1} y_{2} .
\end{array}
$$


Substituting $y_{i} \mapsto x_{i}$ if $i \in\{0, \ldots, 3\}$ and

$$
\begin{aligned}
& y_{4} \mapsto x_{0}-x_{3} \\
& y_{5} \mapsto x_{1}-x_{3} \\
& y_{6} \mapsto x_{2}-x_{3} \\
& y_{7} \mapsto x_{2}-2 x_{3} \\
& y_{8} \mapsto x_{1}-2 x_{3} \\
& y_{9} \mapsto x_{0}-2 x_{3}
\end{aligned}
$$

sends $J_{\Delta} \rightarrow I_{X}$. The simplicial complex $\Delta$ has ten vertices, and is six dimensional, with ten faces of top dimension, and $f$-vector

$$
f(\Delta)=(1,10,45,110,155,126,55,10)
$$

By construction, the specialization by Equation 5 yields the original $G C_{3,2}$ set. However, this is not very satisfying: we knew the specialization would result in a GC set because we reverse engineered it to do so.

The next example is really the punchline of the paper: it shows that specialization can cause non-GC components to become GC components.

Example 4.2. The monomial ideal $J_{\Delta}$ generated by

$\left\langle y_{1} y_{5} y_{6}, y_{2} y_{6} y_{7}, y_{3} y_{7} y_{8}, y_{4} y_{5} y_{8}, y_{1} y_{5} y_{7}, y_{2} y_{6} y_{8}, y_{5} y_{6} y_{7}, y_{5} y_{6} y_{8}, y_{5} y_{7} y_{8}, y_{6} y_{7} y_{8}\right\rangle$

is the Stanley-Reisner ideal for a simplicial complex $\Delta$ on 8 vertices, with

$$
f(\Delta)=(1,8,28,46,35,10)=f\left(\Delta^{\vee}\right) .
$$

A computation shows that 6 of the 10 components are monomial GC; and that $\Delta$ has four maximal monomial hyperplanes: $\left\{y_{5}, y_{6}, y_{7}, y_{8}\right\} . I_{\Delta}$ is codimension three, and specializing yields a $G C_{3,2}$ set, which is a one-lattice.

4.1. Summary. This paper gives a combinatorial recipe for constructing $G C_{d, n}$ sets from simplicial complexes of a special type. By Theorem 2.11 and Theorem 3.12, a quotient of the Stanley-Reisner ideal of a Bi-Cohen Macaulay simplicial complex $\Delta$ of degree $\left(\begin{array}{c}n+d \\ d\end{array}\right)$ and codimension $d$ by a regular sequence is $n$-correct. The GC property is very special, and so in Definition 3.9 we give a monomial version of the GC property. Theorem 3.13 then gives necessary and sufficient conditions for a component $P_{i}$ in the primary decomposition of $I_{\Delta}$ to have the monomial GC property. Theorem 3.12 shows the GC property is preserved under specialization.

A test case are Chung-Yao sets, which we show can be obtained by specializing the $j-1$-skeleton of an $i-1$ simplex; we also show Chung-Yao sets are always determinantal. Example4.2 shows that specialization $y_{i} \mapsto l_{i}$ can yield GC sets even when $I_{\Delta}$ is not monomial GC; it often suffices to start with the weaker condition that $I_{\Delta}$ has many primary components which are monomial GC.

Acknowledgements: We thank an anonymous referee for many helpful suggestions. Computations in the software system Macaulay2 were essential; the package is available at http://www.math.uiuc.edu/Macaulay2. 


\section{REFERENCES}

[1] A. Apozyan, A six dimensional counterexample for the $G M_{d}$ conjecture, Jaen J. Approximation, 3 (2011), 161-172.

[2] A. Apozyan, Conjectures on $G C_{n}$ sets, J. Contemp. Math. Anal., 46 (2011), 69-76.

[3] A. Apozyan, G. Avagyan, G. Ktryan, On the Gasca-Maeztu conjecture in $\mathbb{R}^{3}$, East J. Approximation, 16 (2010), 25-33.

[4] L. Berzolari, Sulla determinazione di una curva o di una superficie algebrica e su alcune questioni di postulazione, Lomb. Ist. Rend., 47 (1914), 556-564.

[5] A. Björner, I. Peeva, J. Sidman, Subspace arrangements defined by products of linear forms, J. London Math Soc., 71 (2005), 273-288.

[6] C. de Boor, Multivariate polynomial interpolation: Conjectures concerning GC-sets, Numer. Algorithms, 45 (2007), 113-125.

[7] C. de Boor, A. Ron, On multivariate polynomial interpolation, Const. Approx., 6, (1990), 287-302.

[8] J. Busch, Lagrange interpolation on $\mathbb{R}^{2}$, Rev. Union. Math. Argen., 36 (1990), 33-38.

[9] J. Carnicer, M. Gasca, A conjecture on multivariate polynomial interpolation, Rev. R. Acad. Cien. Serie A. Mat. 95 (2001), 145-153.

[10] J. Carnicer, M. Gasca, T. Sauer, Interpolation lattices in several variables, Numer. Math., 102 (2006), 559-581.

[11] J. Carnicer, C. Godes, Classification of sets satisfying the geometric characterization, Numer. Algorithms, 50 (2009), 145-154.

[12] K. Chung, T. Yao, On lattices admitting unique Lagrange interpolation, SIAM J. Num. Anal., 14 (1977), 735-743.

[13] J. Eagon, V. Reiner, Resolutions of Stanley-Reisner rings and Alexander duality, J. Pure Appl. Algebra, 130 (1998), 265-275.

[14] S. Eliahou, M. Kervaire, Minimal resolutions of monomial ideals, J. Algebra, 129 (1990), 1-25.

[15] G. Fløystad, J. Vatne, Bi-Cohen-Macaulay simplicial complexes and their associated coherent sheaves, Comm. Algebra, 33 (2005), 3121-3136.

[16] M. Gasca, J. Maeztu, On Lagrange and Hermite interpolation in $\mathbb{R}^{k}$, Numer. Math., 39 (1982), 1-14.

[17] M. Gasca, T. Sauer, On the history of multivariate polynomial interpolation, J. Comput. Appl. Math., 122 (2000), 23-35.

[18] M. Gasca, T. Sauer, Multivariate polynomial interpolation, Advances in Comp. Math., 12 (2000), 377-410.

[19] E. Gorla, Lifting the determinantal property. Algebra, geometry and their interactions, Contemp. Math 448, (2007), 69-89.

[20] H. Hakopian, K. Jetter, G. Zimmermann, A new proof of the Gasca-Maeztu conjecture for $n=4$, Journal of Approximation Theory, 159, (2009), 224-242.

[21] H. Hakopian, K. Jetter, G. Zimmermann, The Gasca-Maeztu conjecture for $n=5$, Numer. Math., 127, (2014), 685-713.

[22] H. Hakopian, L. Refayelyan, On a generalization of the Gasca-Maeztu conjecture, New York J. Mathematics, 21, (2015), 351-367.

[23] I. Peeva, Graded Syzygies, Springer, Berlin-Heidelberg-New York, 2011.

[24] J. Radon, Zur mechanischen Kubatur, Monatsh. Math. 52 (1948) 286-300.

[25] T. Sauer, Y. Xu, On multivariate Lagrange interpolation, Math. Comp. 64 (1995) 1147-1170.

[26] H. Schenck, Computational Algebraic Geometry, Cambridge University Press, (2003).

Fieldsteel: Mathematics Department, University of Illinois Urbana-Champaign, UrBANA, IL 61801, USA

Schenck: Mathematics Department, University of Illinois Urbana-Champaign, UrBANA, IL 61801, USA

E-mail address: fieldst2@math.uiuc.edu

E-mail address: schenck@math.uiuc.edu 